\title{
Comportamiento termico-energético de una vivienda social de la ciudad de Mendoza, Argentina
}

\author{
Thermal-energy efficiency of a social house in the city of \\ Mendoza, Argentina
}

\begin{tabular}{|c|c|}
\hline & $\begin{array}{l}\text { María Victoria Mercado } \\
\text { Alfredo Esteves } \\
\text { Celina Filippín }\end{array}$ \\
\hline & Resumen \\
\hline María Victoria Mercado & $\begin{array}{l}\text { lestado hace entrega del } 30 \% \text { de las viviendas que se habitan por año } \\
\text { en la ciudad de Mendoza, Argentina. El presente trabajo evalúa: (a) la } \\
\text { calidad térmico-energética de una vivienda social, por medio de }\end{array}$ \\
\hline $\begin{array}{r}\text { Laboratorio de Ambiente Humano } \\
\text { y Vivienda }\end{array}$ & $\begin{array}{l}\text { mediciones in-situ en condiciones de uso real; (b) el requerimiento } \\
\text { energético necesario por medio de un balance; (c) la simulación de la vivienda en }\end{array}$ \\
\hline $\begin{array}{r}\text { Centro de Ciencia y Técnica } \\
\text { Consejo Nacional de } \\
\text { Investigaciones Científicas y } \\
\text { Técnicas }\end{array}$ & $\begin{array}{l}\text { el programa SIMEDIF, donde se ajustó el modelo y se ensayaron mejoras de } \\
\text { aplicación fácil y económica; y (d) un sondeo cualitativo de las sensaciones } \\
\text { térmicas. Los resultados mostraron que el edificio presenta un déficit en su }\end{array}$ \\
\hline $\begin{array}{l}\text { Av. Ruiz Leal s/n, Parque General } \\
\text { San Martín (5500), Mendoza - } \\
\text { Argentina }\end{array}$ & $\begin{array}{l}\text { rendimiento térmico-energético: los consumos energéticos reales son menores a } \\
\text { los resultados obtenidos por cálculo. Esto se presume debido a que el usuario se ve }\end{array}$ \\
\hline Tel.: 0054-0261-5244310 & imposibilitado de asumir el costo económico por el consumo energético que \\
\hline $\begin{array}{r}\text { E-mail: } \\
\text { mvmercado@lab.cricyt.edu.ar }\end{array}$ & $\begin{array}{l}\text { supondría mantener la vivienda en condiciones de confort. Las mejoras de la } \\
\text { calidad térmica de la envolvente, simuladas luego de ajustado el modelo, implican } \\
\text { el } 35.6 \% \text { de ahorro de energía auxiliar y el logro de condiciones de confort dentro } \\
\text { de los espacios. }\end{array}$ \\
\hline $\begin{array}{r}\text { Laboratorio de Ambiente Humano } \\
\text { y Vivienda }\end{array}$ & Palabras claves: Rendimiento térmico-energético. Vivienda social. Rehabilitación \\
\hline $\begin{array}{r}\text { Centro de Ciencia y Técnica } \\
\text { Consejo Nacional de } \\
\text { Investigaciones Científicas y }\end{array}$ & Abstract \\
\hline $\begin{array}{r}\text { Técnicas } \\
\text { Tel.: 0054-0261-5244310 }\end{array}$ & The state provides $30 \%$ of houses per year in the city of Mendoza. This study \\
\hline E-mail: & $\begin{array}{l}\text { evaluates: (a) the thermal-energy quality of a social house, through in-situ } \\
\text { measurements under conditions of actual use; }(b) \text { needed energy requirements } \\
\text { through a balance; }(c) \text { a simulation of the house using the SIMEDIF program, in } \\
\text { which the model was adjusted, and some easy to implement and economical }\end{array}$ \\
\hline Celina Filippín & improvements were tested; and (d) a qualitative survey of thermal sensations. The \\
\hline INTA Santa Rosa La Pampa & results indicated that the building has a deficit in its thermal and energy efficiency: \\
\hline $\begin{array}{r}\text { Consejo Nacional de } \\
\text { Investigaciones Científicas y } \\
\text { Técnicas }\end{array}$ & $\begin{array}{l}\text { real energy consumption is lower than the results obtained by calculation. This is } \\
\text { presumably because the user is unable to bear the financial cost of the energy }\end{array}$ \\
\hline Tel.: 0054-0261-5244310 & consumption that would keep the house in a comfort situation. Improvements in the \\
\hline E-mail: <cfilippin@cpenet.com.ar & $\begin{array}{l}\text { thermal quality of the envelope, which were considered in the simulation, result in } \\
35.6 \% \text { of energy savings and the achievement of comfort conditions in the housing } \\
\text { spaces. }\end{array}$ \\
\hline $\begin{array}{r}\text { Recebido em 20/02/2010 } \\
\text { Aceito em 02/06/2010 }\end{array}$ & Thermal-energy efficiency. Social housing. Bioclimatic rehabilitation. \\
\hline
\end{tabular}




\section{Introducción}

\section{Vivienda social y energía}

En términos amplios, la vivienda no sólo es "el techo", sino un sistema que incluye: el terreno, la infraestructura, el equipamiento socialcomunitario; esto según una localización y dentro de un contexto cultural, económico, político y tecnológico determinado.

La vivienda social es aquélla destinada al mejoramiento de la situación habitacional de personas o grupos que no tienen la posibilidad de encarar económicamente la construcción de su vivienda en forma individual. Por esta razón, el estado se convierte en el principal sujeto de acción para proporcionar, financiar y organizar la entrega de viviendas a esta franja de la población.

Es así que el estado hace entrega de un importante porcentaje de las viviendas que se habitan por año en la ciudad de Mendoza (IPV, 2001). En estos hogares, es ineludible otorgar condiciones de calidad térmica que propicien el confort de sus habitantes sin el excesivo consumo de energías de base fósil, ya que por sus costos, resulta muy difícil de pagar. Una adecuada envolvente, que logre un armonioso diálogo con el clima del lugar y un diseño bioclimático para un correcto comportamiento térmico-energético se hace necesario e imperioso de implementar en las viviendas actuales y futuras de construir.

Las condiciones de habitabilidad brindadas por las viviendas financiadas por el Instituto Provincial de la Vivienda (IPV) de la provincia de Mendoza de la República Argentina - ente encargado de gerenciar la construcción de la vivienda social en Argentina-, afectan a un número importante de personas. El aspecto térmico no debería ser desconsiderado en ningún tipo de construcción, especialmente en los emprendimientos de vivienda del Estado, debido a que el gasto de funcionamiento y mantenimiento que generan las mismas posteriormente resultan significativos, considerando el presupuesto familiar disponible de la mayoría de los usuarios (MARTINEZ, 2005).

En la República Argentina el sector residencial es el encargado del 53\% del consumo energético nacional, del cual se utiliza el $58 \%$ para calefaccionar las viviendas (EVANS, 2005). Las crisis energéticas sufridas han provocado un incremento tarifario, percibido en los últimos años. Como una consecuencia de esto, los niveles de confort bajaron en los sectores con menor poder adquisitivo, debido a la incapacidad de asumir económicamente esta situación (MERCADO; ESTEVES, 2004). Al mismo tiempo, aparece en escena el subsidio económico que ejerce el estado sobre el gas natural, que no permite que la población de clase media alta y los mismos organismos del estado perciban claramente la compleja situación de disponibilidad y uso desmedido del recurso (DE DICCO, 2006). En el país, se accede al gas natural con un costo hasta veinticinco veces menor en relación a otros lugares de Latinoamérica como Santiago de Chile, Río de Janeiro o Montevideo (Tabla 1). Estos valores indican que el costo energético seguirá ascendiendo en los años venideros, lo que dará como consecuencia un empeoramiento de la situación de confort de la franja poblacional de menores recursos.

Consecuentemente, ante la situación energéticoeconómica planteada que padece el país, y ambiental que atraviesa la humanidad, es primordial que el estado construya viviendas energéticamente eficientes y que propicien ambientes térmicamente confortables.

\begin{tabular}{l|c}
\hline \multicolumn{1}{c|}{ Ciudad } & Costo por $\mathbf{~ m}^{3} /$ gas natural [US\$] ${ }^{4}$ \\
\hline Mendoza - Argentina & $0.062^{1}$ \\
\hline La Pampa - Argentina & $0.097^{2}$ \\
\hline Bariloche - Argentina & $0.007^{3}$ \\
\hline Río de Janeiro - Brasil & $2.618^{2}$ \\
\hline Santiago - Chile & $1.018^{2}$ \\
\hline Montevideo-Uruguay & $0.582^{2}$ \\
\hline
\end{tabular}

\footnotetext{
${ }^{1}$ Tasa de acuerdo a facturación en 2010, ECOGAS CUYANA (facturación bimensual Empresa EROGAS CUYANA).

${ }^{2}$ Tasa de acuerdo a facturación en 2009, CAMUZZI Gas del Sur (facturación bimensual Empresa CAMUZZI Gas del Sur).

${ }^{3}$ Alejandro D. González (2009).

${ }^{4}$ Cotización dólar a día 15 de febrero de 2010.

Tabla 1 - Costo residencial de gas natural por $\mathrm{m}^{3}$ en US\$
} 


\section{Situación térmica habitacional}

La provincia de Mendoza tenía hacia el 2001 un déficit habitacional de 100.000 viviendas, de las cuales 30.000 se agrupa en la necesidad de viviendas nuevas (IPV, 2001), para las cuales es importante y urgente diseñar con una nueva conciencia energética las edificaciones sociales. Sin embargo ya existe un parque habitacional de vivienda social que conforma el $30 \%$ del total de viviendas de la ciudad de Mendoza, con una marcada condición de disconfort térmico y poca salubridad (MERCADO; ESTEVES; FILIPPÍN, 2007). También en ellas se debe pensar para mejorar la calidad de vida de sus ocupantes en el marco del desarrollo sustentable.

Algunos de los indicadores que definen el déficit de viviendas están determinados por la situación de hacinamiento y el mal estado de conservación del parque habitacional existente. Se observa que, en ninguno de estos considerandos para la elaboración de los censos, se cuenta o evalúa la calidad térmica de la vivienda ni en forma global, ni de su envolvente. Esto es preocupante debido a que una envolvente de mala calidad térmica asegura condiciones de disconfort, lo que conlleva a problemas de salubridad. Esta situación resulta muy común en las viviendas nuevas y de las 2 últimas décadas. Numerosos autores advierten este fenómeno (CZAJKOWSKI, 2000; MITCHELL, 2005; MARTINEZ, 2004; MITCHELL; ESTEVES, 2004).

\section{Calidad térmica de la envolvente}

"Los edificios son barreras a la lluvia, al viento y, a veces, filtros sutiles a la luz y calor. Rodeados de entornos variables, donde cambian el día y la noche, el calor y el frío, el viento y la calma, la lluvia y el sol; se convierten en refugios de artificiales condiciones, como islas de tranquilidad en un mundo incómodo" (SERRA, 2000).

En el año 1970 se comenzó a utilizar el término de "envolvente" refiriéndose a un límite entre un ambiente exterior y uno interior en la arquitectura En el año 1972, Pat O’Sullivan comienza a trabajar con la envolvente como concepto de filtro climático (GANEM, 2006).

La vivienda es un conjunto de sistemas que interactúan entre sí para dar lugar a espacios habitables con determinadas y diferentes condiciones. La envolvente, como parte del sistema juega un rol principal en la ganancia y pérdida de calor. Es el elemento que materializa "un filtro selectivo" para crear microclimas internos que le propicien confort al usuario. Esta piel edilicia debe ser lo suficientemente estudiada para garantizarle al usuario: (a) protección de las condiciones ambientales exteriores (frío-calor excesivos, lluvia, vientos);

(b) resguardo socio-cultural, seguridad, privacidad;

(c) posibilidad de apropiación por medio de la transformación individual de cada usuario.

De Rosa et al. (1989) estudiaron tres tipologías de vivienda de la ciudad de Mendoza y concluyeron en su trabajo que "desde el punto de vista del consumo energético la situación de disconfort constituye una constante". En la Tabla 2 se muestra los resultados del estudio cualitativo, realizado por los autores. La evaluación fue realizada en veinte viviendas de diferentes tipologías (compactas, quebradas y duplex), a través de encuestas en las que se debía ponderar de 0 a 3 , el indicador cualitativo de sensación térmica.

Si se observan los resultados de la Tabla 2, indican que son más los casos en que la vivienda no es confortable $(53,79$ y $62 \%$ en el caso de las viviendas compactas, quebradas y tipo dúplex, respectivamente) en caso de compactas, $79 \%$ las quebradas y $62 \%$ los duplex). Es posible inferir que es debido a la baja calidad térmica de la envolvente y a la ausencia de un proyecto de vivienda térmica y energéticamente eficiente.

Las normas argentinas, específicamente la serie IRAM 11600, trata el tema de la calidad térmica de los edificios construidos en territorio nacional y del consumo energético de ellos, estableciendo los métodos de cálculo y los valores mínimos de sus condiciones higrotérmicas. La norma 11605 (IRAM, 1996), específicamente, otorga tres niveles de transmitancia que corresponden a un grado decreciente de la calidad térmica tanto en los paramentos verticales como en las cubiertas, siendo: nivel A: recomendado, nivel B: medio y nivel C: mínimo. Ver Tabla 3 (MERCADO; ESTEVES, 2004)

El Instituto Provincial de la Vivienda de Mendoza (IPV), construye las viviendas sociales sin tener en cuenta las normas IRAM. Por lo que el resultado de la realidad construida es equivalente a menos del nivel C. Considerando que las viviendas sociales de la ciudad de Mendoza son térmicamente precarias la pregunta es: ¿por qué no aplicar el nivel A? Este nivel estima un importante espesor de aislación a incorporar en el paramento, esto dificulta la implementación desde el aspecto práctico y económico. Debido a estas consideraciones sobre el uso de la norma 11605, el trabajo de Mercado y Esteves (2004), proponen un cuarto nivel (Figura 1), orientado a unificar tres aspectos en cuanto a la incorporación de aislación térmica: el térmico, el práctico y el económico. 


\begin{tabular}{l|c|c}
\hline \multicolumn{1}{c|}{ Tipología } & Sensación térmica agradable & Sensación térmica fría o muy fría \\
\hline Compactas $^{1}$ & 0.47 & 0.53 \\
\hline Quebradas $^{1}$ & 0.21 & 0.79 \\
\hline Duplex $^{1}$ & 0.38 & 0.63 \\
\hline vivienda IPV -invierno $^{2}$ & 0.1 & 0.9 \\
\hline vivienda IPV - verano $^{2}$ & 0.9 & 0.1 \\
\hline
\end{tabular}

1: PID No 3-094000/88-dir: Carlos de Rosa (DE ROSA et al., 1989).

2 :Datos obtenidos de las encuestas realizadas (2007).

Tabla 2 - Resultados de los estudios cualitativos según encuestas

\begin{tabular}{c|c|c|c|c|c|c}
\hline \multirow{2}{*}{$\begin{array}{c}\text { Temperatura } \\
\text { exterior de } \\
\text { diseño }\end{array}$} & \multicolumn{2}{|c|}{ Nivel A $\left[\mathbf{K}=\mathbf{W} / \mathbf{m}^{2} \mathbf{K}^{\mathbf{0}}\right]$} & \multicolumn{2}{c}{ Nivel B $\left[\mathbf{K}=\mathbf{W} / \mathbf{m} 2 \mathbf{K C}^{\mathbf{0}}\right]$} & \multicolumn{2}{c}{ Nivel C $\left[\mathbf{K}=\mathbf{W}^{\prime} \mathbf{m} \mathbf{K}^{\mathbf{0}}\right]$} \\
\cline { 2 - 7 } & Muros & Techos & Muros & Techos & Muros & Techos \\
\hline-15 & 0.23 & 0.20 & 0.60 & 0.52 & 1.01 & 1.00 \\
\hline-14 & 0.23 & 0.20 & 0.61 & 0.53 & 1.04 & 1.00 \\
\hline-13 & 0.24 & 0.21 & 0.63 & 0.55 & 1.08 & 1.00 \\
\hline-12 & 0.25 & 0.21 & 0.65 & 0.56 & 1.11 & 1.00 \\
\hline-11 & 0.25 & 0.22 & 0.67 & 0.58 & 1.15 & 1.00 \\
\hline-10 & 0.26 & 0.23 & 0.69 & 0.60 & 1.19 & 1.00 \\
\hline-9 & 0.27 & 0.23 & 0.72 & 0.61 & 1.23 & 1.00 \\
\hline-8 & 0.28 & 0.24 & 0.74 & 0.63 & 1.28 & 1.00 \\
\hline-7 & 0.29 & 0.25 & 0.77 & 0.65 & 1.33 & 1.00 \\
\hline-6 & 0.30 & 0.25 & 0.80 & 0.67 & 1.39 & 1.00 \\
\hline-5 & 0.31 & 0.27 & 0.83 & 0.69 & 1.45 & 1.00 \\
\hline-4 & 0.32 & 0.28 & 0.87 & 0.72 & 1.52 & 1.00 \\
\hline-3 & 0.33 & 0.29 & 0.91 & 0.74 & 1.59 & 1.00 \\
\hline-2 & 0.35 & 0.30 & 0.95 & 0.77 & 1.67 & 1.00 \\
\hline-1 & 0.36 & 0.31 & 0.99 & 0.80 & 1.75 & 1.00 \\
\hline 0 & 0.38 & 0.32 & 1.00 & 0.83 & 1.85 & 1.00 \\
\hline
\end{tabular}

Tabla 3 - Niveles de transmitancia establecidos por la norma IRAM 11605

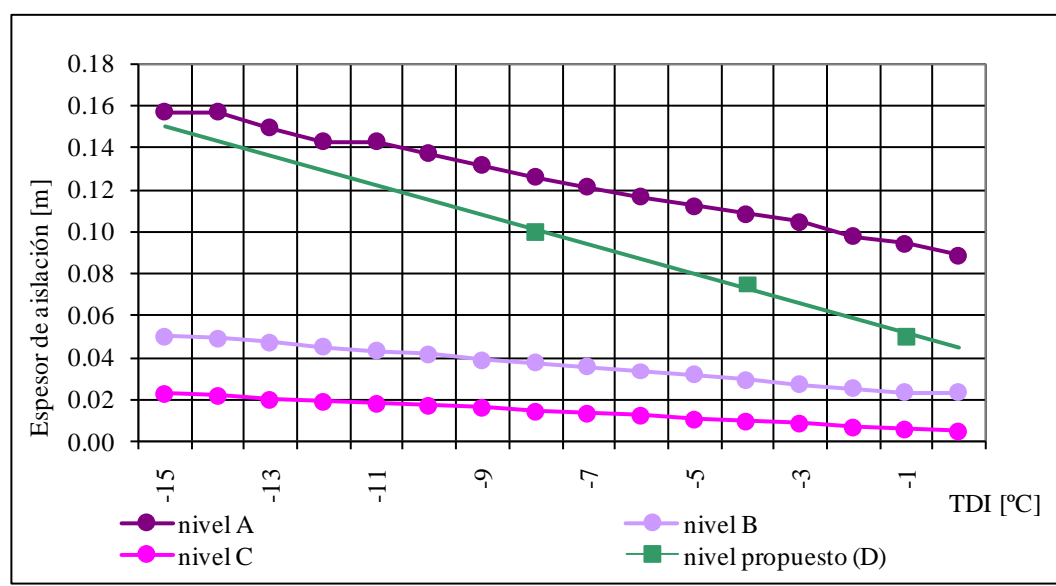

Figura 1 - Espesores de aislación térmica para cumplimentar con los diferentes niveles de transmitancia establecidos por la norma IRAM 11605 más un cuarto nivel que se propone en el trabajo Mercado y Esteves (2004)

Como respuesta al aspecto térmico, el nuevo nivel propuesto por los autores otorga, de acuerdo a la zona, condiciones de confort y minimiza las pérdidas innecesarias de energía por medio de la conservación. El práctico, en la ejecución de la obra, no supone la incorporación de mano de obra especializada y es de fácil aplicación. Y por último el aspecto económico, supone una inversión cercana al $0.08 \%$ del total de la obra ${ }^{1}$.

\footnotetext{
${ }^{1}$ En relación a valores de obra del año 2007.
} 
La calidad de la envolvente es tan solo uno de los componentes del sistema que involucra un edificio. $\mathrm{Si}$ se considera al edificio como un sistema integrado al que se le incorporan determinadas tecnologías para mejorar la calidad "térmica", reacciona todo el sistema, obteniendo como resultado un ahorro de energía, que genera una disminución de los costos operativos y, la amortización de la inversión que origina su implementación.

Con el objetivo de conocer el funcionamiento térmico-energético del sistema de una vivienda se procede a realizar una evaluación cuantitativa. Conjuntamente como parte del sistema se contempla también los factores sociales de la misma por medio de encuestas que completa la evaluación cualitativa global.

Por último, se utiliza el programa de simulación SIMEDIF (FLORES et al., 2000), para evaluar mejoras que se puedan realizar a la vivienda, con el objetivo de alcanzar condiciones de confort sin el uso desmedido del recurso agotable: gas natural.

\section{Comportamiento termo- energetico de la vivienda de estudio}

\section{Lugar y clima}

La provincia de Mendoza tiene un territorio de $148.827 \mathrm{~km}^{2}$ y se encuentra situada en el centrooeste de la República Argentina sobre una zona semidesértica de clima árido. Los tres oasis (norte, este y sur) rigen el crecimiento y la distribución poblacional sobre el territorio. La ciudad de Mendoza se ubica en el centro del Oasis norte $\left(32^{\circ}\right.$ $40^{\prime}$ latitud sur y $68^{\circ} 51^{\prime}$ longitud oeste; a una altura sobre nivel del mar de $750 \mathrm{~m}$ promedio). Figura 2.

La zona presenta características de tierra mediterránea, de clima árido a semiárido, con una fuerte amplitud térmica, En la Figura 3 se observa la temperatura máxima absoluta (TMAA), temperatura media TMM, y la temperatura mínima absoluta (TMIA). Las precipitaciones anuales $(218 \mathrm{~mm})$ son reducidas con un mayor porcentaje de incidencia en la época estival. Otra característica fundamental es la intensa radiación solar, con un cielo marcadamente sin nubes en la mayor parte del año (65\%). Estas condiciones resultan apropiadas para incorporar a las viviendas sistemas solares pasivos de calefacción.

La ciudad de Mendoza es el conglomerado urbano más importante de la provincia, denominado "Gran Mendoza", que alberga el $64 \%$ de la población, (885.151 habitantes [INDEC, 2001]) y ocupa el $11 \%$ del territorio jurisdiccional de Mendoza. Desde la situación demográfica-urbana es posible entender la gran demanda de viviendas nuevas (cerca de 30000; IPV Mza) que tiene la provincia a pesar de la continua, pero insuficiente, construcción de barrios nuevos.

\section{Monitoreo térmico de la vivienda social plan FONAVI}

Con el objetivo de analizar el funcionamiento termo-energético de la vivienda plan FONAVI, se ha trabajado con un caso de estudio. El mismo es una vivienda construida en el año 2006 en el departamento de Las Heras del barrio denominado "12 de Octubre" que se encuentra sobre la periferia de la ciudad de Mendoza, llegando al pie de monte de la cordillera de Los Andes. La ejecución de la obra estuvo a cargo de una empresa constructora subcontratada por el Instituto Provincial de la Vivienda (IPV Mendoza) quien realizó la inspección técnica.

En la primer etapa de estudio se realizó el monitoreo térmico de la vivienda para avalar las condiciones de invierno y verano. Para ello se registraron los valores de temperatura interior de las áreas funcionales de la vivienda (cocinacomedor y dos dormitorios), la temperatura exterior y la radiación solar sobre plano horizontal. La frecuencia de mediciones de temperatura fue cada 15 minutos y se utilizaron dataloggers tipo HOBO, el sensor de radiación solar es un solarímetro Kipp y Zonen modelo CM5.

La segunda etapa consistió en realizar la evaluación energética de la misma de acuerdo a un balance térmico (ESTEVES; GELARDI, 2003), con el objetivo de conocer el consumo energético anual mediante la fracción de ahorro solar (FAS).

Se tienen también datos relevados de los usuarios en cuanto a consumo energético de gas natural y electricidad. 


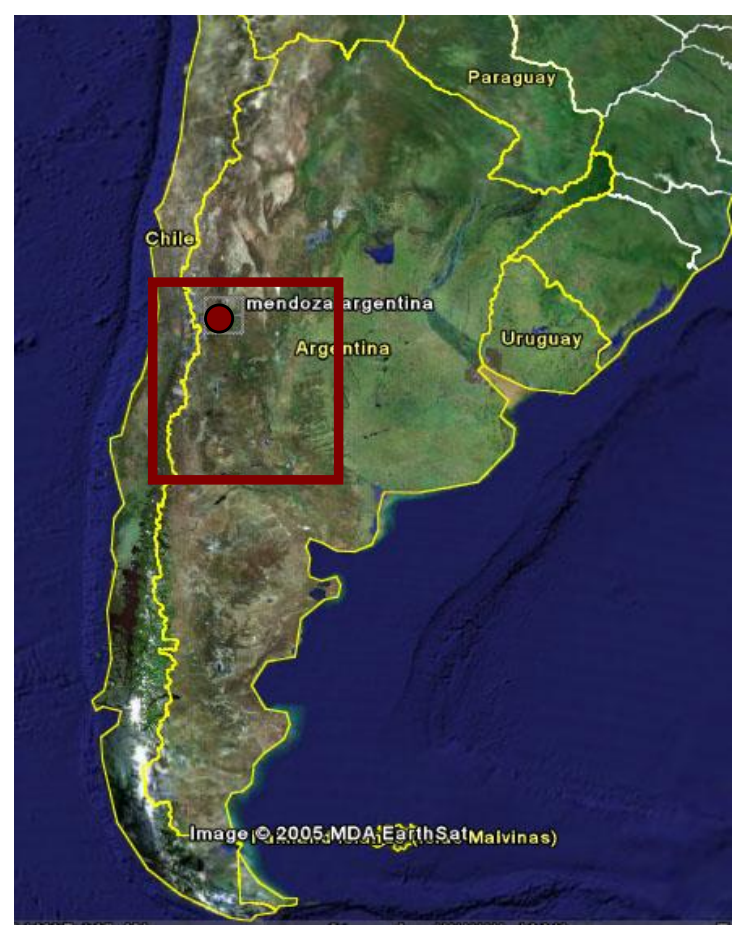

Figura 2 - Ubicación de la ciudad de Mendoza en la República Argentina

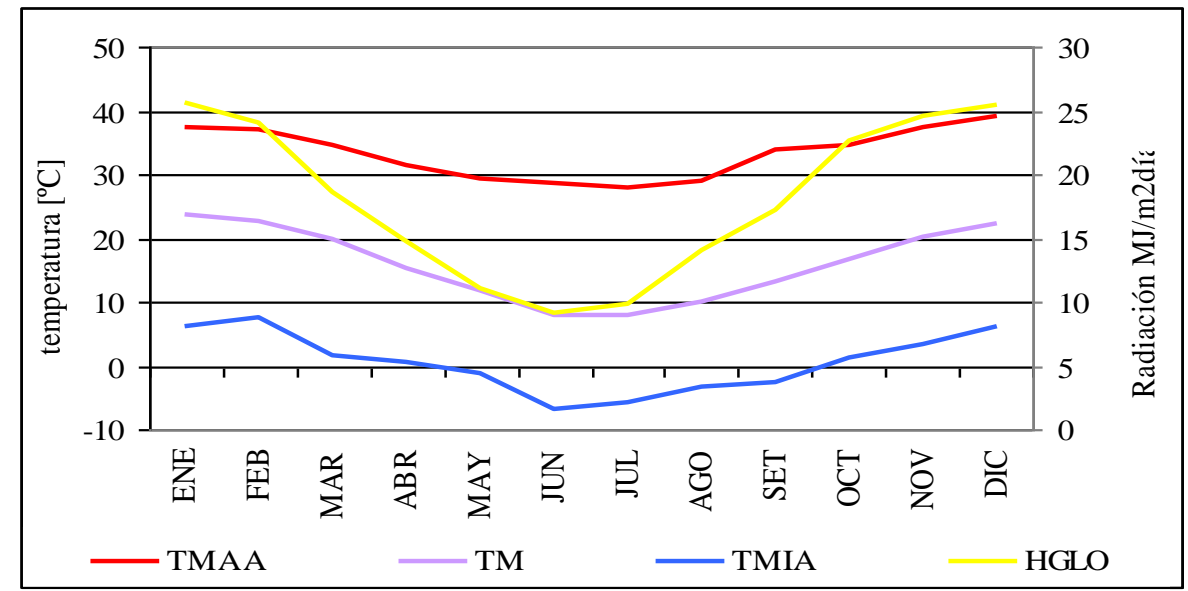

Figura 3 - Temperaturas medias y absolutas mensuales, radiación solar global mensual

\section{La vivienda}

El mencionado barrio 12 de octubre, fue seleccionado por tratarse de un barrio comprendido en el programa FONAVI (Fondo Nacional para la Vivienda), que mantiene un esquema funcional y un sistema constructivo actual y repetitivo de la vivienda social construida en de este plan.

El sistema constructivo se compone de: muros de ladrillón macizo común $(\mathrm{K}=2.41 \mathrm{~W} / \mathrm{m} 2)$ con espesor de $0.20 \mathrm{~m}$; cubierta de techo liviana inclinada, compuesta por machimbre de pino del lado interior, poliestireno expandido como aislamiento térmico de $2,5 \mathrm{~cm}$ de espesor y chapa como protección exterior $(\mathrm{K}=1.10 \mathrm{~W} / \mathrm{m} 2)$; aberturas con marcos y hojas de chapa de acero plegada y ventanas con un solo vidrio. Cuenta con una superficie cubierta de $63 \mathrm{~m} 2$ en donde se resuelve un espacio diurno único (cocinacomedor), un baño y dos dormitorios, con la posibilidad de ampliar la vivienda hacia el fondo de la misma con un dormitorio más. En la Figura 4 se puede observar la planta y una fotografía de la vivienda monitoreada.

La forma se resuelve en dos cuerpos prismáticos yuxtapuestos. La envolvente resulta de $146.46 \mathrm{~m}^{2}$, donde el $57.2 \%$ corresponde a muros y el $42.8 \%$ a techos. El FAEP (factor de área envolvente-piso) es de 2.3, es decir la vivienda tiene $2.3 \mathrm{~m}^{2}$ de envolvente por $\mathrm{m}^{2}$ de superficie cubierta. Esto último indica que la resolución formal de la vivienda podría 
ser más compacta y consecuentemente eficiente energéticamente, por ejemplo una alternativa es modificar, en el diseño original, la altura de los techos inclinados, cuya cumbrera se ubica a $3.5 \mathrm{~m}$. Esto permitiría además de reducir el FAEP, reducir los costos de cada vivienda, en consecuencia los costos de todos un barrio.

\section{Mediciones: situación de Invierno}

Los registros se tomaron durante catorce días completos, desde el 14 al 28 de agosto de 2007. Para el análisis se selecciona un período comprendido entre el 24 al 28. En la figura 5, se presentan los datos obtenidos de la radiación sobre plano horizontal y la temperatura del aire exterior e interior para los tres locales medidos.

La temperatura mínima exterior oscila entre $1.6^{\circ} \mathrm{C}$ y $3.3^{\circ} \mathrm{C}$ y la máxima entre $10.2^{\circ} \mathrm{C}$ y $17.5^{\circ} \mathrm{C}$ (amplitud térmica exterior $10-15^{\circ} \mathrm{C}$ ). La temperatura del aire interior presenta una amplitud térmica $12^{\circ} \mathrm{C}$ promedio en el espacio diurno, con temperaturas mínimas cercanas a $\operatorname{los} 11^{\circ} \mathrm{C}$ y máxima interior por encima de $\operatorname{los} 26^{\circ} \mathrm{C}$ ). Esto evidencia la falta de confort en la vivienda y el permanente intercambio de calor entre interior y exterior. Sin embargo esta diferencia se reduce a $7^{\circ} \mathrm{C}$ en el área de los dormitorios, por tratarse del sector menos calefaccionado por medio del uso de energías convencionales.

La Figura 5 permite observar además, una diversidad en el comportamiento térmico de la vivienda entre el espacio diurno y el espacio de dormitorios. La temperatura del primero oscila entre $25-30^{\circ} \mathrm{C}$ cuando los dormitorios se mantienen entre $15-18^{\circ} \mathrm{C}$. Esto implica una diferencia de la temperatura entre $10^{\circ}$ y $12^{\circ} \mathrm{C}$ entre un local y los otros, situación que genera sensación de disconfort $\mathrm{y}$, a veces situaciones de riesgo en la salud de los usuarios.

Realizando una confrontación entre los dos sectores diferenciados anteriormente, se observa un gradiente en la amplitud térmica que ocurre en los espacios de dormitorios. La amplitud térmica que se presenta en el espacio diurno es de $15^{\circ} \mathrm{C}$, mientras que en el espacio nocturno desciende a $9^{\circ} \mathrm{C}$. Es posible inferir que la fluctuación de la temperatura se debe a la falta de calefacción durante el día (menor ganancia) y a la falta de conservación energética que posee el edificio (mayores pérdidas).

Las temperaturas alcanzadas durante la mañana, en el espacio diurno, no alcanzan la temperatura mínima de confort $\left(18^{\circ} \mathrm{C}\right)$. Hacia la tarde y por la noche la situación es diferente, debido a que se supera la temperatura máxima $\left(26^{\circ} \mathrm{C}\right)$. Consecuentemente, la casa permanece unas pocas horas en el rango de confort.

Por otro lado, los dormitorios no alcanzan condiciones térmicas de confort en la mayor parte del día. Los días 24 y 25 de agosto, donde permanecen unas pocas horas de la noche por encima de $18^{\circ} \mathrm{C}$, se observa la existencia de sobrecalentamiento en el espacio diurno, debido al uso extremo de calefacción.

Los espacios de cocina-comedor, son espacios pequeños, donde los individuos tienen una fuerte influencia térmica de la envolvente (intercambio por radiación desde las superficies que los rodean). A partir de esta apreciación se considera que el usuario, inclusive con alta temperatura del aire, siente frío por la baja temperatura de las paredes como consecuencia de una alta transferencia de calor, a razón de esto su respuesta es seguir haciendo uso del artefacto de calefacción con el que dispone para mitigar esta condición de disconfort-térmico constante.

Se observa que si bien la masa térmica de la vivienda es suficientemente importante, a través de la tecnología de muros de ladrillón macizo, la elevada amplitud indica una calidad térmica insuficiente de techo para mantener las condiciones mínimas de estabilidad térmica. La falta de aislación en muros y el escaso espesor utilizado en techos genera pérdidas energéticas en el invierno que podrían disminuir aumentando aquél de acuerdo a las normativas correspondientes, más aún tratándose de viviendas construidas por el Estado Nacional.

\section{Mediciones: situación de Verano}

En la estación de verano se tomó como periodo de medición una semana completa, desde el 21 al 28 de diciembre de 2007. Para el estudio de los resultados se seleccionaron cinco días: desde el 21 al 25. En la Figura 6, se presentan los registros de radiación solar sobre plano horizontal y los de la temperatura del aire exterior e interior de cada local medido.

La radiación solar sobre plano horizontal no superó los $550 \mathrm{~W} / \mathrm{m}^{2}$. La temperatura mínima exterior osciló entre los 22 y los $24^{\circ} \mathrm{C}$ par los días con cielo claro. Para el día con cielo parcialmente nublado el valor desciende a $16^{\circ} \mathrm{C}$. Por otro lado, las temperaturas máximas se sitúan sobre $\operatorname{los} 35^{\circ} \mathrm{C}$, siendo la excepción el día 25 que registra un valor cercano a $\operatorname{los} 30^{\circ} \mathrm{C}$. 

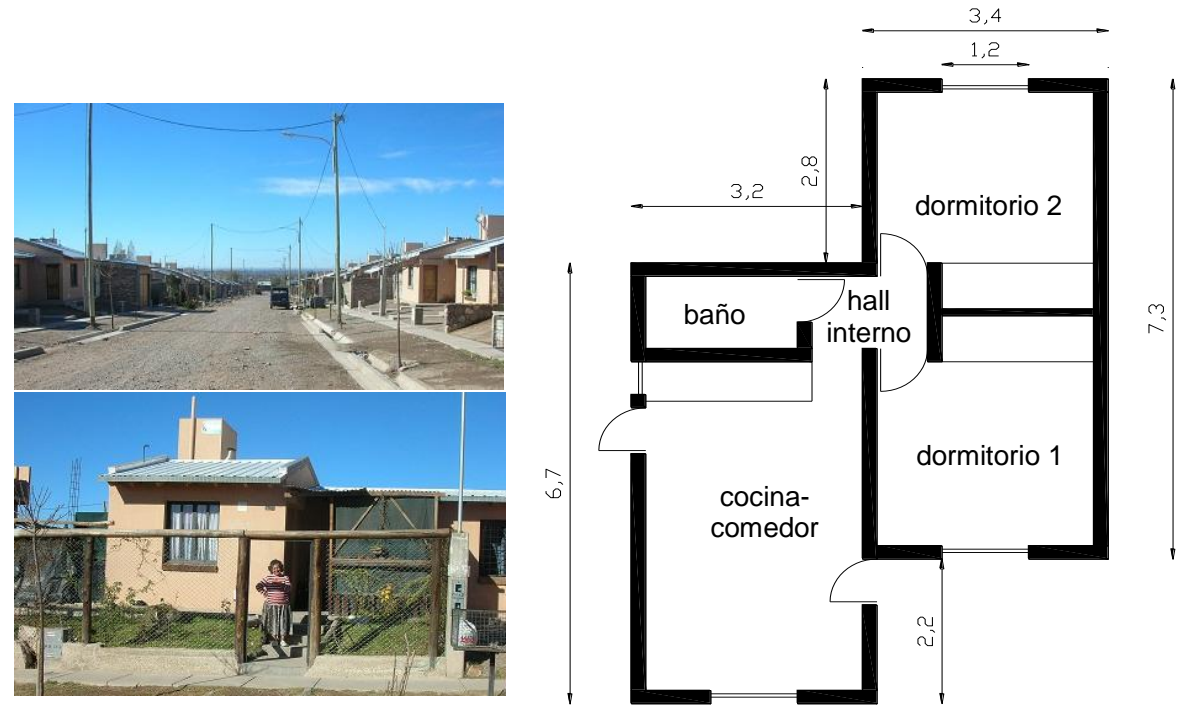

Figura 4 - Planta y fotografía de vivienda $B^{\circ} 12$ de octubre, tipología FONAVI

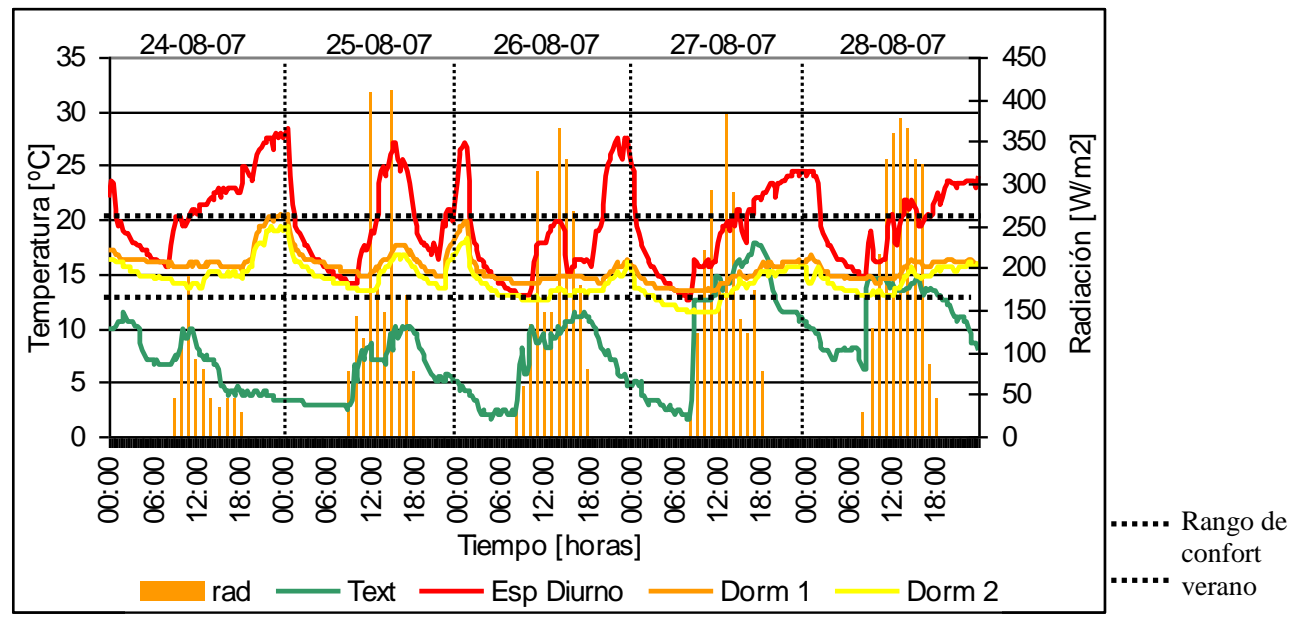

Figura 5 - Radiación solar y temperaturas de aire interior y exterior para los días 24 a 28 de agosto de 2007

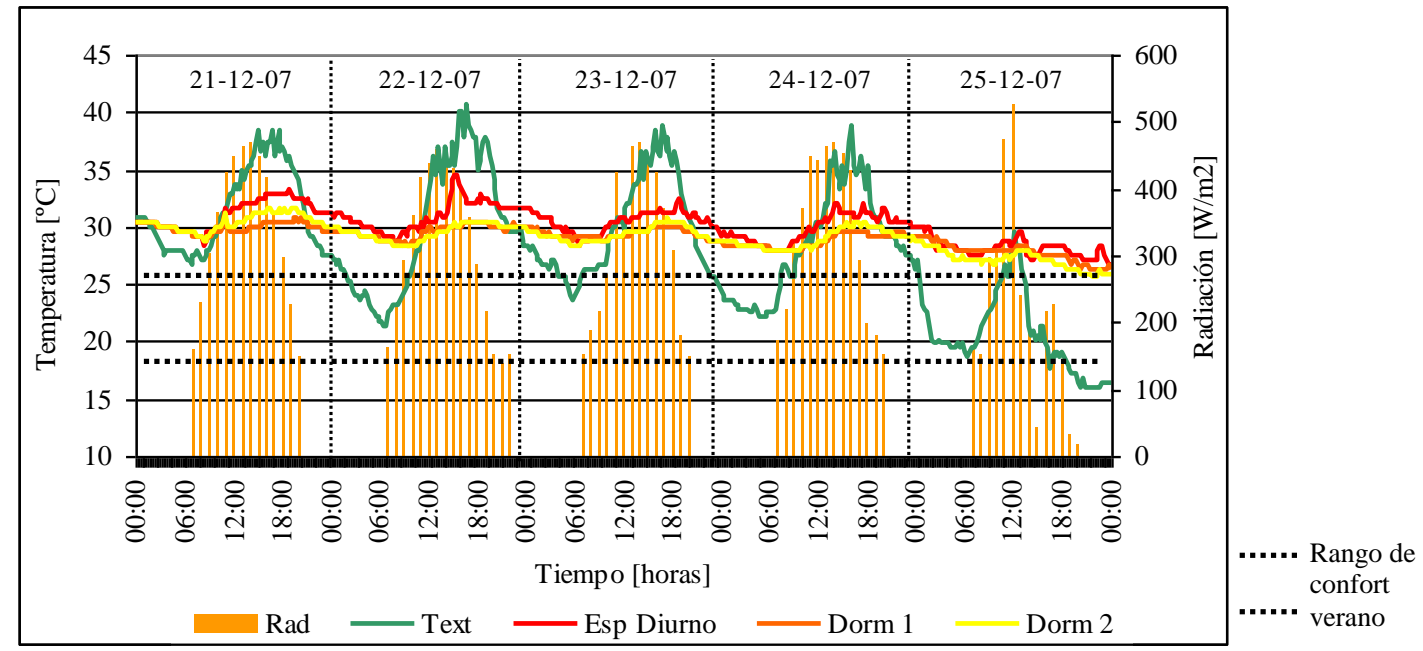

Figura 6 - Radiación solar y temperaturas de aire interior y exterior para los días 21 a 25 de diciembre de 2007 
Las temperaturas interiores de la vivienda (espacio diurno y dormitorios), estuvieron diariamente fuera del rango de confort para verano $\left(27^{\circ} \mathrm{C}\right.$ a $\left.33^{\circ} \mathrm{C}\right)$. $\mathrm{La}$ amplitud térmica interior y exterior fue de $6^{\circ} \mathrm{C}$ y $20^{\circ} \mathrm{C}$, respectivamente.

Las temperaturas internas muestran muy baja variabilidad debido, fundamentalmente, a que no hubo ventilación nocturna, que permitiría enfriar la masa térmica lamiendo el calor acumulado durante el día. Esta situación genera altas temperaturas permanente y riesgos sanitarios a los que se encuentran sometidos los usuarios.

\section{Evaluación energética}

Se realizó la evaluación energética de la vivienda a través del programa computacional de balance térmico para el estudio de la optimización de un diseño arquitectónico con estrategias bioclimáticas, el cual permite integrar las distintas variables que se conjugan para dar un comportamiento térmico final eficiente del edificio. El programa hace uso del método de la relación carga térmica/colector y lo combina con el método Quadri para el cálculo de la potencia de calefacción (ESTEVES; GELARDI, 2003).

En la Tabla 4 se presentan los valores del coeficiente neto de pérdidas CNP total y disgregado de acuerdo al elemento constructivo en porcentaje de incidencia sobre el total (muros, techo, fundaciones, infiltraciones y aberturas). Se observa también la fracción de ahorro solar en porcentaje (FAS) (BALCOMB et al., 1982), el consumo necesario anual para mantener a la vivienda en confort $\left(\mathrm{m}^{3}\right.$ de gas natural) y la potencia necesaria a instalar en la vivienda (Kcal/h).

A partir de las mediciones in-situ de la vivienda, se estimó, una mala calidad de la envolvente. Por medio del balance energético y la disgregación del coeficiente neto de pérdidas es posible aseverar este supuesto, teniendo en cuenta que por muros y techos se produce el $71.4 \%$ de la fuga energética del edificio.

La fracción de ahorro solar de $0.9 \%$, muestra la falta de un diseño bioclimático y de consideración de las orientaciones en el proyecto de la vivienda. Este valor podría ascender al $10 \%$ si se contemplara un diseño acorde con la localización geográfica y sus condiciones climáticas (MERCADO, et al. 2008). Esta variable es muy significativa, ya que con igual superficie cubierta, pero con una correcta ubicación y un adecuado dimensionamiento de las áreas transparentes, se podría alcanzar un ahorro energético del orden del $36 \%$.

Observando los valores de consumo anual de gas natural $\left(1395,7 \mathrm{~m}^{3}\right)$ necesario para mantener las mínimas condiciones de confort térmico (Temperatura interior $=20^{\circ} \mathrm{C}$ ) es fácil advertir que es excesivo al tratarse de una vivienda de $60 \mathrm{~m}^{2}$. Esto supone $23.3 \mathrm{~m}^{3} / \mathrm{m}^{2}$.año frente al consumo de una casa bioclimática con un consumo de $6.5 \mathrm{~m}^{3} /$ $\mathrm{m}^{2}$.año (ESTEVES; MERCADO; GANEM, 2006).

Sin embargo, al considerar los valores de consumo en las boletas de consumo de servicio (Figura 7), el consumo anual real de gas natural es el $58 \%$ del consumo anual para mantener la vivienda en confort que otorga el balance térmico (Tabla 4). Esto establece dos consideraciones:

(a) el nivel de confort, como se ha visto en las mediciones, no es alcanzado inclusive con el gran consumo de energía convencional (gas natural);

(b) el usuario se ve imposibilitado para hacer frente al costo económico por el consumo energético, que supondría mantener la vivienda en condiciones de confort.

\begin{tabular}{l|c}
\hline \multicolumn{1}{c|}{ Item } & Vivienda FONAVI \\
\hline CNP total [W/ $\left.{ }^{\circ} \mathrm{C}\right]$ & 361.9 \\
\hline CNP muros [\%] & 52.3 \\
\hline CNP techos [\%] & 19.1 \\
\hline CNP fundaciones [\%] & 11 \\
\hline CNP infiltraciones [\%] & 15.7 \\
\hline CNP aberturas [\%] & 1.9 \\
\hline FAS [\%] & 0.9 \\
\hline Calor Auxiliar Anual [Kwh/año] & 15102 \\
\hline Consumo Anual, gas natural [m3] & 1395.7 \\
\hline Potencia Necesaria[Kcal /h] & 5224 \\
\hline
\end{tabular}

Tabla 4 - Resultados del balance energético para una vivienda FONAVI 
De acuerdo al valor estipulado por el programa del balance para la potencia necesaria, es posible dimensionar los artefactos de calefacción requeridos por este tipo de vivienda. Siendo de $5224 \mathrm{kcal}$, con un calefactor de 3000 kcal más otro artefacto pequeño bastaría.

\section{Simulación térmica de la vivienda y presentación de mejoras}

Se ingresó el modelo de la vivienda en el programa de simulación SIMEDIF para Windows (FLORES et al., 2000), y se ajustaron las variables con datos medidos en la estación de verano, situación más favorable para poder calibrar el modelo debido a la inexistencia de ganancia interna por efecto del encendido de los calefactores. Posteriormente, se simuló la estación de invierno y se evaluó el comportamiento térmico de la vivienda a partir de la implementación de medidas de mejoramiento que implicaran, el menor costo económico posible.

El ingreso de datos al programa se realizó de la siguiente manera: la cocina comedor (denominado espacio diurno), los dormitorios (Dorm 1 con orientación norte y Dorm 2 con orientación sur), el hall interno y el baño se ingresaron como cinco locales por separado, para el período de 14 días seguidos en la estación de invierno y verano correspondientes a los períodos medidos. Todos los cerramientos se consideraron como muros de ladrillo macizo de la zona, con un coeficiente de conducción de $2.41 \mathrm{~W} /\left(\mathrm{m}^{2}{ }^{\circ} \mathrm{C}\right)$, para los techos se consideró un coeficiente de $1.1 \mathrm{~W} / \mathrm{m}^{2}{ }^{\circ} \mathrm{C}$. Como coeficientes convectivos externos se empleó un valor de $10.38 \mathrm{~W} / \mathrm{m}^{2}{ }^{\circ} \mathrm{C}$ para muros que reciben radiación solar. Los coeficientes convectivos interiores se fijaron en $5.8 \mathrm{~W} /\left(\mathrm{m}^{2}{ }^{\circ} \mathrm{C}\right)$ para superficies verticales y $9 \mathrm{~W} /\left(\mathrm{m}^{2}{ }^{\circ} \mathrm{C}\right)$ para superficie de techo. El número de renovaciones de aire por hora de cada local se fijó en 1.

En la Figura 8 se presentan los datos medidos y datos simulados para cuatro días del período de verano para el espacio diurno, además se presenta el coeficiente de determinación para medir la relación de ajuste resultando $\mathrm{R}^{2}=0.91 \quad(\mathrm{R}=0.95$, muestra que existe una probabilidad menor a 0.05 de que los resultados hayan sido obtenidos al azar).

Las medidas de mejoramiento térmico simuladas se establecieron bajo la premisa de priorizar el bajo costo de aplicación:

(a) protección de las áreas transparentes durante el verano a través de cortinas exteriores e interiores, reduciendo el coeficiente de conductividad de $5.8 \mathrm{~W} / \mathrm{m}^{2} \mathrm{C}$ a $3.4 \mathrm{~W} / \mathrm{m}^{2} \mathrm{C}$ durante las horas de mayor radiación solar; (b) ventilación cruzada aprovechando las aberturas de la vivienda;

(c) reducción de la radiación incidente sobre los muros este por medio de pérgolas verdes perennes que permitan el acceso del sol en la estación de invierno. En la región es frecuente la utilización de la vid en los patios de los hogares;

(d) aislación térmica en muros de un espesor de $0.05 \mathrm{~m}$ de poliestireno expandido y el incremento de $0.025 \mathrm{~m}$ del mismo material en techos, alterando los coeficientes de transmitancia en $0.74 \mathrm{~W} / \mathrm{m}^{2} \mathrm{C}$ y $0.64 \mathrm{~W} / \mathrm{m}^{2} \mathrm{C}$ respectivamente.

En la Figura 9 se presentan los resultados de la simulación realizada para los días seleccionados del período estival, del 21 al 24 de diciembre de 2007. Se observa que se logra la diminución de la amplitud térmica interior en el orden de $4-5^{\circ} \mathrm{C}$, además de anular la asimetría radiante entre locales interiores a la vivienda. Asi mismo se logra que la temperatura interior de los locales decienda en un orden de $7^{\circ} \mathrm{C}$ para el espacio diurno y $3^{\circ} \mathrm{C}$ para los dormitorios. Considerando el rango de confort para verano de $20-26^{\circ} \mathrm{C}$, la vivienda alcanza las condiciones necesarias para brindarles a sus ocupantes confort térmico durante la época de verano, con altas temperaturas exteriores.

Los resultados alcanzados para el período de invierno se exponen en la Figura 10. Se consideraron los datos obtenidos para cinco días consecutivos desde el 24 de agosto de 2007.

Al igual que las condiciones de verano, disminuye la zonificación térmica dentro de la vivienda con una amortiguación de las temperaturas interiores y, una variación entre local diurno y locales nocturnos de $3^{\circ} \mathrm{C}$, tratándose de un valor admisible.

El espacio diurno se mantiene dentro del rango de confort con la incidencia de la ganancia solar a través de las áreas transparentes, y considerando además la ganancia de calor aportada por un calefactor de $3000 \mathrm{kcal}$ ubicado en el hall interno. $\mathrm{Si}$ a esto se le suma algún otro elemento que aporte una ganancia de calor mínima dentro del local (como por ejemplo una hornalla de cocina al mínimo $=600 \mathrm{~W}$ ) la temperatura alcanzada permitiría estar en condiciones de confort. Por otro lado las habitaciones presentan un comportamiento más severo. Sin embargo, si se considera una temperatura de $15^{\circ} \mathrm{C}$, permitida para la situación, con un mayor nivel de (2.2 cló), (OLGYAY, 2008), se estaría también en presencia de una situación aceptable en relación a las condiciones exteriores extremas. 


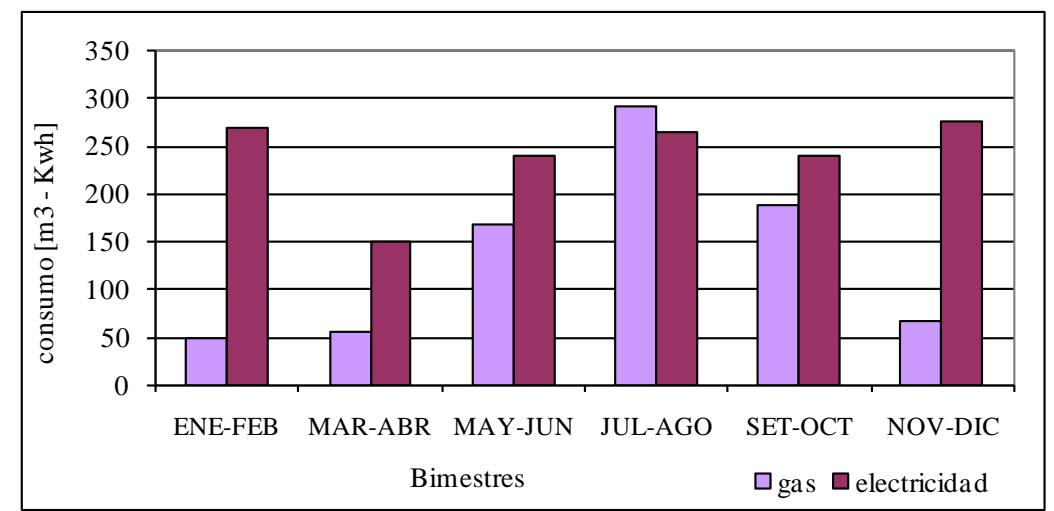

Figura 7 - Consumos energéticos en gas natural $\left[\mathrm{m}^{3}\right]$ y electricidad $[\mathrm{Kwh}]$
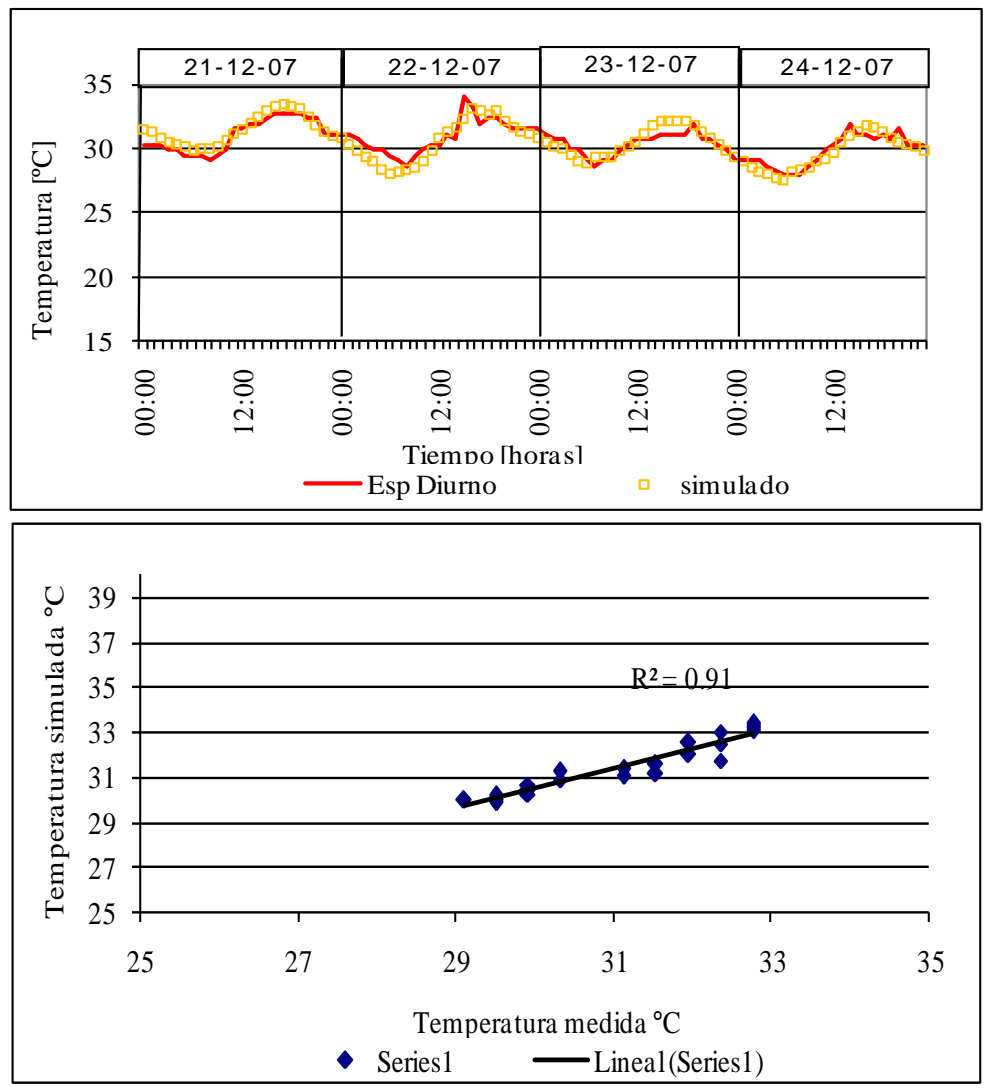

Figura 8 - Correlación entre datos medidos y datos simulados, para la evaluación del ajuste del modelo

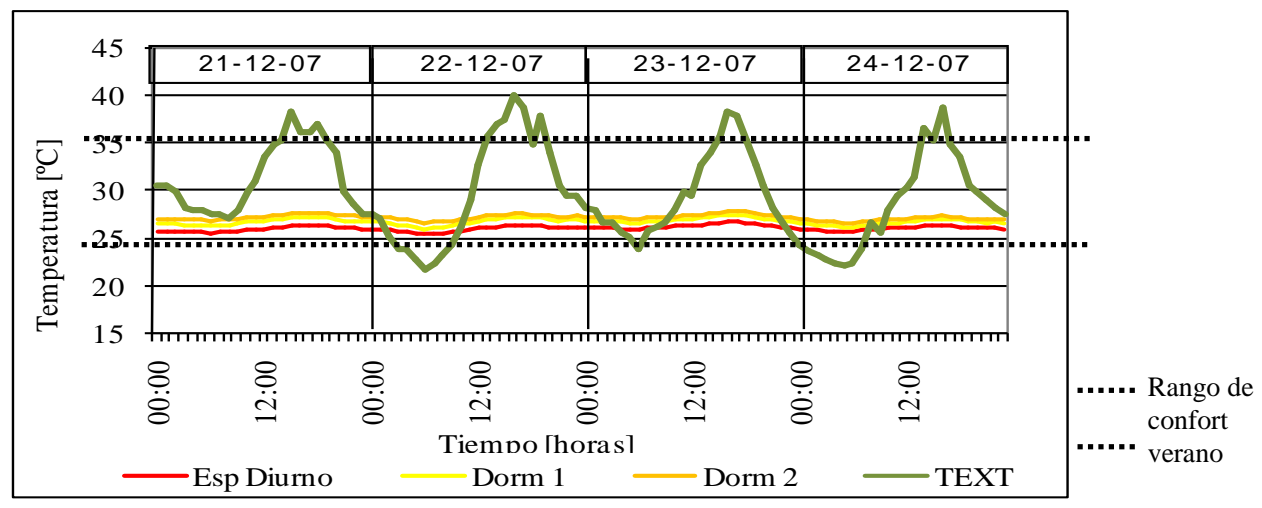

Figura 9 - Temperaturas de aire interior simuladas para un período de 4 días de la estación de verano 


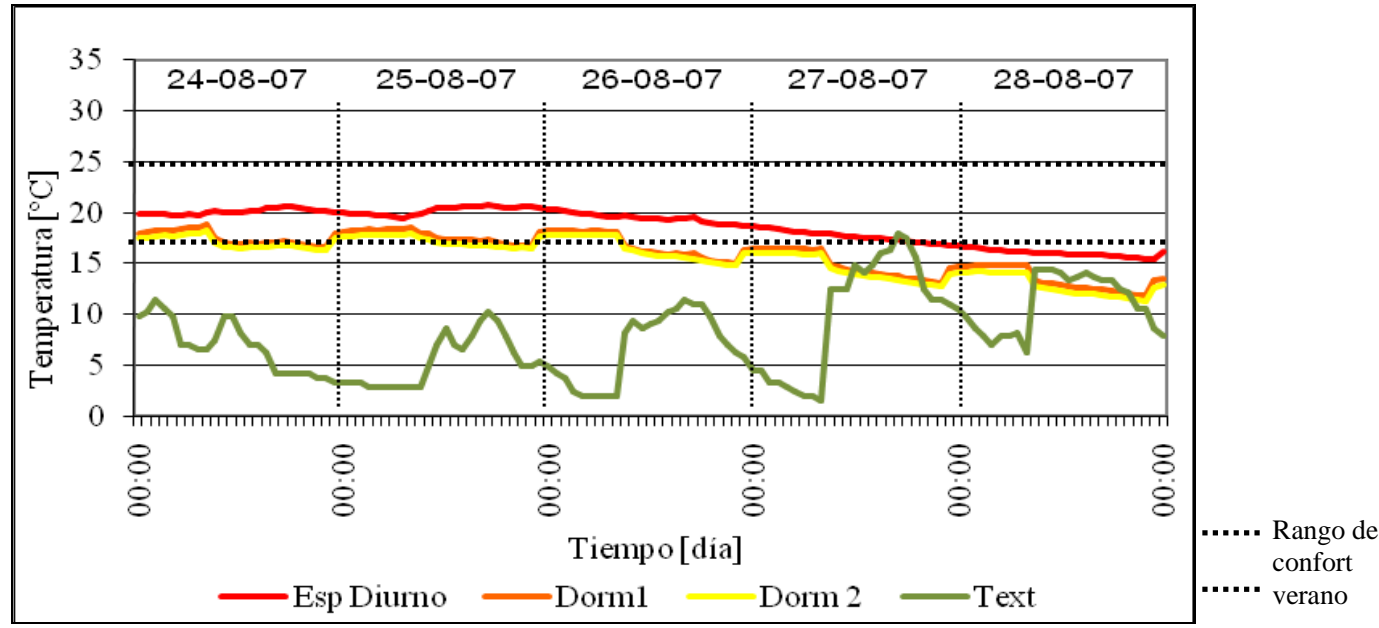

Figura 10 - Temperaturas de aire interior simuladas para un período de 5 días de la estación de invierno
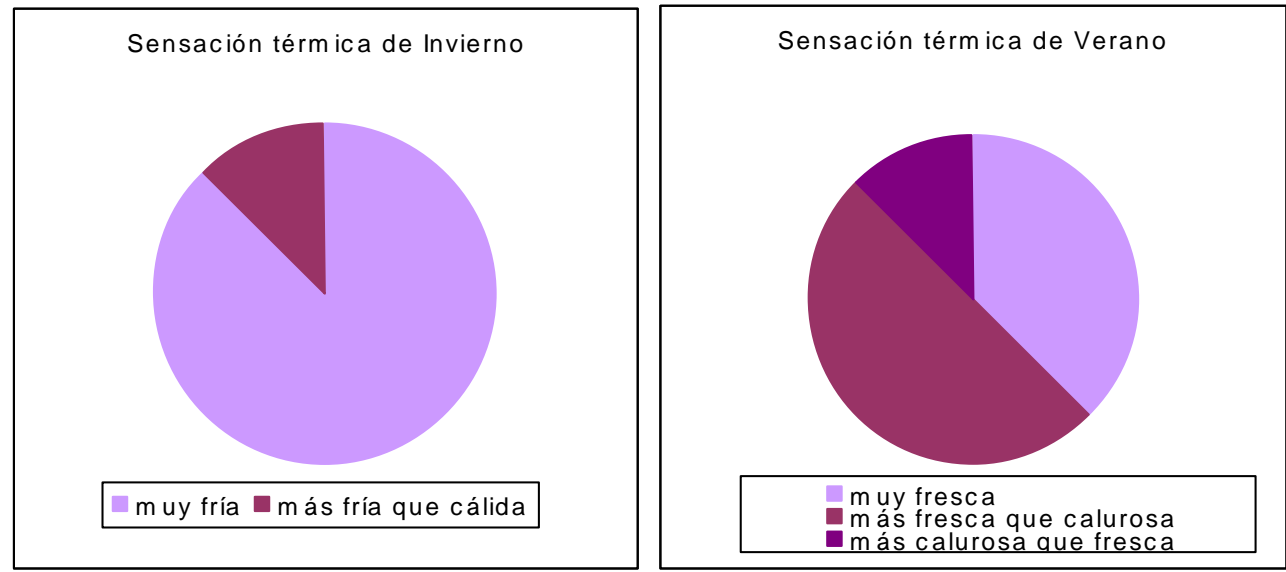

Figura 11 - Resultados de encuesta, Ítems: sensación térmica en invierno y verano de los usuarios

\section{Evaluación cualitativa: encuestas}

A partir de encuestas realizadas a los usuarios de las viviendas que conforman el barrio $12 \mathrm{de}$ Octubre, se analiza la información obtenida con el propósito de adquirir datos sobre su percepción térmica, lo que se define en este trabajo como evaluación cualitativa.

La encuesta realizada se dividió en cuatro partes, una primera parte de datos generales, una segunda de evaluación térmica para invierno y verano, la tercera de iluminación y por último unas preguntas sencillas ahondando en la predisposición de los habitantes de realizar modificaciones en la vivienda para acrecentar las condiciones de confort dentro de la vivienda. En el presente trabajo se analizan las variantes térmicas únicamente.

De las treinta y dos viviendas del barrio se encuestó un $25 \%$ del grupo, de este grupo, la mitad orientadas hacia el sur y la segunda mitad orientadas hacia el norte, debido a que el barrio se organiza en el eje este-oeste, dejando los accesos de las viviendas hacia el norte y/o sur.

\section{Resultados: situación General}

Las viviendas cuentan con una ocupación media de 5.5 ocupantes. Estos permanecen la mayor parte del tiempo diario en el espacio destinado a cocinacomedor o espacio diurno como se lo ha nombrado anteriormente.

\section{Resultados: situación Térmica}

Se consultó el conocimiento de la existencia de aislación térmica en la vivienda. Existen usuarios que poseen conceptos erróneos o mala información. El $20 \%$ consideran al ruberoid (cartón embreado) como aislación térmica, otro $30 \%$ no tiene conocimiento si la construcción tiene aislación y el $50 \%$ restante es conciente que no posee este elemento.

El $90 \%$ de los encuestados perciben sus viviendas muy frías en invierno. En la estación de verano la sensación es diversa, el $50 \%$ siente la residencia más fresca que calurosa, el $40 \%$ muy fresca y el $10 \%$ más calurosa que fresca (Figura 11). 
Es posible inferir que las sensaciones indicadas para verano, son el resultado de dos situaciones: ala ubicación del barrio sobre la primer planicie del pie de monte de la precordillera de Los Andes, donde las brisas son más frecuentes y más frescas que en el centro de la ciudad, b- la altura de la vivienda, al tener $3.5 \mathrm{~m}$ de altura en un espacio reducido de $15,0 \mathrm{~m}^{2}$, el aire caliente se mantiene por estratificación por encima del nivel de percepción de los usuarios $(1,6 \mathrm{~m})$.

En cuanto al instrumento y tiempo de utilización de los sistemas de calefacción, el $10 \%$ usa sólo la cocina (hornallas, debido a que el encendido del horno supone un costo mayor que no pueden afrontar), el $60 \%$ utiliza cocina y pantallas infrarrojas y el $30 \%$ restante ha podido adquirir calefactores convectivos convencionales. En la mayoría de las viviendas el sistema que se utiliza como calefacción permanece encendido todo el día, un porcentaje del $90 \%$. El 10\% manifiesta que hace uso del mismo, seis horas por día.

Respecto a los sistemas de acondicionamiento de verano, el $10 \%$ de las casas poseen sistemas de ventilación mecánica (ventiladores) haciendo uso también del enfriamiento convectivo nocturno. El $90 \%$ restante utiliza sólo el enfriamiento convectivo nocturno. De estas últimas el $57 \%$ manifiesta que mantiene abiertas las ventanas todo el día, un $13 \% 6$ horas durante la mañana y el $30 \%$ durante la noche.

\section{Resultados: transformaciones en la vivienda}

Cuando se habla de realizar remodelaciones en la vivienda que mejoren el estado de la misma, el $80 \%$ está dispuesto a realizarlas por autoconstrucción. De este porcentaje, el $100 \%$ indica que la motivación para querer realizarlo es la búsqueda de mayor bienestar térmico. Para esto, el $84 \%$ de las personas le dedicarían cuatro horas, en un día de la semana. El resto le destinaría ocho horas, divididas en dos días de la semana.

\section{Conclusiones}

Se presenta un estudio térmico exhaustivo realizado en una vivienda de un barrio a través de mediciones en situación de verano e invierno y encuestas a un porcentaje significativo de un barrio típico de la ciudad de Mendoza, construido por el IPV provincial. Las encuestas permiten tomar en cuenta la situación de las personas que viven en este barrio y que son representativos de los barrios de la zona que construye el Organismo.

La vivienda evaluada posee un marcado déficit en su calidad térmica que obliga al usuario a vivir en condiciones térmicas precarias, a sostener un gran costo energético durante la vida útil de la vivienda y por el diseño arquitectónico se lo priva de la posibilidad de hacer un uso racional de la energía.

La calidad térmica de la envolvente de la vivienda social tipo FONAVI, ya sea a través de evaluaciones cuantitativas como cualitativas, es muy deficitaria. A partir de estos resultados se presenta una propuesta de mejoramiento, de bajo costo, que permite mejorar sustancialmente la situación térmica y, generar un grado de confort y salubridad interior deseable.

Con respecto a la situación térmica se puede observar que buscando las mejoras en la calidad térmica de su propio ambiente interior, las personas buscan medios de calefacción a su alcance, utilizan la cocina como primer recurso, pantallas infrarrojas adicionales y cuando pueden (sólo el 30\%) accede a la compra de calefactores. Se observan que las medidas de conservación de energía y aprovechamiento energético solar permiten alcanzar porcentajes de ahorro cercanos al $30 \%$ y reducir, así, la necesidad de potencia de calefacción.

La masa térmica interior que posee la construcción tradicional sirve para mantener las condiciones térmicas y evitar el enfriamiento excesivo en los períodos de no calefacción (durante las noches o cuando los ocupantes salen a trabajar diariamente) (ROAF, 2003). Como se puede observar la cantidad es suficiente, sin embargo, se encuentra mal utilizada al no contar con estrategias de conservación de energía.

Un buen uso de los elementos de la vivienda, como: a- ventilación nocturna para verano y/o b- la utilización de cortinas exteriores para invierno, resuelven parte de las pérdidas, a bajo costo, por los elementos translúcidos.

Una reducción de la altura de la cumbrera, sin afectar el escurrimiento de las escasas precipitaciones del lugar permitiría por un lado, reducir el FAEP y el costo total de la envolvente vertical, por otro, reducir el volumen a calefaccionar. Esta disminución no debería afectar el comportamiento de verano y la estratificación generada por la densidad del aire.

\section{Referências bibliográficas}

CZAJKOWSKI, J. Desarrollo de un Modelo de Ahorro de Energía en Edificios de Vivienda y Determinación de Valores Límite de Calidad Térmica para la República Argentina. Avances en Energías Renovables y Medio Ambiente, Salta, v. 4, n. 2, p. 1.39-1.45, 2000. 
DE ROSA, C. et al. Proyecto de Investigación y Desarrollo $N^{\mathbf{0}}$ 09400/88. Vivienda Social: déficit habitacional y habitabilidad higrotérmica. evaluación y propuestas para su comportamiento en la provincia de Mendoza”. Mendoza, 1989. Documento Inédito.

DE DICCO, R. A. Argentina: aumenta la demanda de hidrocarburos y caen las reservas. Buenos Aires: IDICSO, 2006. (Serie Artículos de opinión y Breves Informes).

ESTEVES, A.; GELARDI, D. Docencia en Arquitectura Sustentable: programa de optimización de proyectos de arquitectura en el balance térmico. Avances en Energías

Renovables y Medio Ambiente, Salta, v. 7, n. 2, p. 10.31-10.34, 2003.

ESTEVES A, MERCADO M. V, GANEM C. Reciclaje Solar Pasivo de una Vivienda en el Centro-Oeste de Argentina. Avances en Energías Renovables y Medio Ambiente, Salta, v. 10, n.1, p. 5.37-5.42, 2006.

\section{EVANS, J. M. Energía en el Hábitat}

Construido: panorama en Argentina. Buenos Aires. Ed. Univesitarias UBA, 2005.

GANEM, C. Rehabilitación Ambiental de la Envolvente de Viviendas: el caso de Mendoza, Argentina. 2006. Tesis (Doctoral) - Universidad Politécnica de Cataluña, Cataluña, 2006.

GONZALEZ A. D. Energy Subsidies in Argentina Lead to Very Low Termal Efficiency: posible way out trrough building improvement policies.

Energies, v. 2, p. 769-788, 2009.

FLORES, S. F. et al. SIMEDIF para Windows. Salta: INENCO, 2000. Disponible en: $<$ http://inenco.unsa.edu.ar/ seflores/>. Acceso en: 15 feb 2005.

INSTITUTO ARGENTINO DE

NORMALIZACIÓN Y CERTIFICACIÓN

(IRAM). IRAM 11605: aislamiento térmico de edificios, condiciones de habitabilidad en viviendas". Buenos Aires, 1996.

INSTITUTO PROVINCIAL DE LA VIVIENDA (IPV). Déficit Habitacional en la Provincia de Mendoza. Mendoza, 2001. Disponible en: <http://www.ipvmendoza.gov.ar>. Acceso en: 11 ago 2008.
BALCOMB, J. D. et al. Passive Solar Design Handbook. Boulder: American Solar Energy Society, 1982.

MARTÍNEZ, C. Evaluación Cualitativa de Condiciones Ambientales de Viviendas del IPV en S. M. de Tucumán. Avances en Energías Renovables y Medio Ambiente, Salta, v. 8, n.1, p. 05.01-05.06, 2004.

MARTÍNEZ, C. Comportamiento TérmicoEnergético de Envolvente de Vivienda en San Miguel de Tucumán en Relación a la Adecuación Climática. Avances en Energías Renovables y Medio Ambiente, Salta, v. 9, , p. 05.01-05.06, 2005.

MERCADO, M. V.; ESTEVES, A. Arquitectura Sustentable: estudio térmico y técnico económico de la incorporación de aislación térmica. Energías Renovables y Medio Ambiente, Salta, v. 15, p. 45-52, 2004.

MERCADO, M. V.; ESTEVES, A.; FILIPPÍN, C. Sistema de Climatización Solar Pasivo: su integración en viviendas de bajo costo. Avances en Energías Renovables y Medio Ambiente, Salta, v. 11, p. 05.121-05.126, 2007.

MERCADO, M. V. et al. Evaluación TérmicoEnergético y Cualitativa de Condiciones Ambientales de una Vivienda Social de la Ciudad de Mendoza. Avances en Energías Renovables y Medio Ambiente, Salta, v. 12, p. 05.73-05.80, 2008.

MITCHELL, J. A.; ESTEVES, A. Diagnóstico de Consumos Energéticos de un Asentamiento Rural del Oasis Norte de Mendoza. Avances en Energías Renovables y Medio Ambiente, Salta, v. 8, n. 1, p. 07.43-07.48, 2004.

MITCHELL, J. A. Consumo de Energía para Calefacción en Avances en Energías Renovables y Medio Ambiente, Salta, v. 9, p. 07.07-07.12, 2005.

OLGYAY, V. Arquitectura y Clima. Barcelona: Gilli, 2008

ROAF, S. Ecohouse 2. London: Architectural, 2003.

SERRA, R. Arquitectura y Climas. 2. ed. Barcelona: Gili, 2000. 